\section{Diferenciais intra-urbanos de vulnerabilidade da população idosa}

\author{
Intra-urban differences in vulnerability among the \\ elderly population
}

\author{
1 Programa de Pós-graduação \\ em Saúde Pública \\ Universidade Federal de \\ Minas Gerais, Belo Horizonte, \\ Brasil. \\ 2 Department of Nutrition, \\ Food Studies \& Public Health, \\ New York University, New \\ York, USA. \\ 3 Departamento de \\ Estatística, Universidade \\ Federal de Minas Gerais, \\ Belo Horizonte, Brasil. \\ 4 Núcleo de Estudos \\ em Saúde Pública e \\ Envelhecimento, Fundação \\ Oswaldo Cruz/Universidad \\ Federal de Minas Gerais, \\ Belo Horizonte, Brasil. \\ Correspondência \\ L. S. Braga \\ Programa de Pós-graduação \\ em Saúde Pública, \\ Universidade Federal de \\ Minas Gerais. \\ Rua Doutor Rubens \\ Guimarães 50, apto. 101, \\ Belo Horizonte, $M G$ \\ 30840-430, Brasil. \\ lusouzabraga@yahoo.com.br
}

\section{Abstract}

This study examined the adequacy of a health vulnerability index (HVI) for identifying intraurban differences in vulnerability in the elderly population. Study participants $(n=1,055)$ were selected by probabilistic sampling of residents in the city of Belo Horizonte, Minas Gerais State, Brazil, aged 60 years or older (mean $=70.1$ years). The HVI was constructed with indicators of socioeconomic conditions and premature mortality at the census tract level. Based on its location and HVI value, each participant's household was classified as low, medium, or high-risk. Results of multivariate ordinal logistic regression models showed graded and statistically significant as sociations between the household's risk level and the social and physical environment, health conditions and behaviors, and health service needs (adjusted prevalence ratios ranging from 1.17; 95\%CI: 1.02-1.34 to 1.65; 95\%CI: 1.45-1.88). The HVI appears to be a useful instrument for identifying health and social service needs among the elderly.

Health Vulnerability; Urban Health; Aged; Health Services
Luciana de Souza Braga ${ }^{1}$

James Macinko 2

Fernando Augusto Proietti 1

Cibele Comini César 1,3

Maria Fernanda Lima-Costa 1,4

\section{Introdução}

O envelhecimento populacional e a urbanização são tendências mundiais neste início de século, com projeções crescentes de ambos os fenômenos, sobretudo em países de média renda 1,2. O rápido envelhecimento populacional em grandes centros urbanos gera preocupação quanto à capacidade dos sistemas sociais e de saúde para atender essa nova demanda. Assim, a identificação de áreas em grandes cidades com maior concentração de idosos vulneráveis e a garantia da distribuição adequada e eqüitativa dos serviços de saúde para essas populações constituem desafios para os sistemas de saúde.

A vulnerabilidade pode ser definida como a capacidade de um indivíduo ou sistema sofrer dano em resposta a um estímulo ${ }^{3}$. Os idosos, como grupo populacional com maior necessidade em saúde, são particularmente vulneráveis às mudanças nas condições que determinam o "envelhecimento ativo". Esse conceito, segundo a Organização Mundial da Saúde (OMS) 1, é definido por seis dimensões: (1) determinantes econômicos (renda, trabalho e proteção social); (2) determinantes sociais (escolaridade, apoio social e segurança); (3) ambiente físico (moradia e vizinhança, ausência de obstáculos ambientais e acesso à água limpa, ar puro e alimentos saudáveis); (4) determinantes individuais (fatores biológicos, genéticos e psicológicos); (5) determinantes comportamentais (comportamentos em 
saúde e participação no cuidado com a própria saúde); (6) serviços sociais e de saúde (promoção da saúde e prevenção de doenças, serviços curativos e assistência social). Cultura e gênero, por influenciarem os demais determinantes, alocam-se transversalmente neste modelo.

Belo Horizonte, capital do Estado de Minas Gerais, é uma das maiores cidades brasileiras com cerca de 2,4 milhões de habitantes, dos quais aproximadamente $10 \%$ são idosos 4 . Embora apresente o quarto maior Produto Interno Bruto do país e alto Índice de Desenvolvimento Humano $(0,839){ }^{4}$, a distribuição da riqueza, da escolaridade e das condições de saúde entre seus habitantes apresenta grandes disparidades 5 . Entre 1998 e 2003, a Secretaria Municipal de Saúde de Belo Horizonte (SMS-BH) desenvolveu um Índice de Vulnerabilidade à Saúde (IVS) para orientar o planejamento das suas ações 5 . O IVS é baseado nas condições socioeconômicas dos setores censitários e em indicadores da mortalidade precoce. As áreas de maior vulnerabilidade (maior IVS) foram prioritárias para a implementação de algumas ações, entre elas, a Estratégia Saúde da Família 6 .

O presente trabalho tem por objetivo examinar a adequação do IVS, antes mencionado, para identificar diferenciais intra-urbanos de vulnerabilidade da população idosa.

\section{Métodos}

\section{Fontes de dados}

Para este trabalho foram utilizadas duas fontes de dados. A primeira foi aquela desenvolvida pela SMS-BH, contendo o IVS de cada setor censitário do município no ano de 2003 5. A segunda foi o Inquérito de Saúde da Região Metropolitana de Belo Horizonte, conduzido entre maio e julho de 20037 .

\section{Índice de Vulnerabilidade à Saúde (IVS)}

Trata-se de um indicador do setor censitário que soma informações sobre cinco componentes: (1) condições de saneamento (esgotamento, abastecimento de água e destino do lixo), (2) habitação (proporção de domicílios improvisados e razão de moradores por domicílio), (3) escolaridade (percentual de analfabetos e de chefes de família com menos de 4 anos de escolaridade completa), (4) renda (percentual de chefes de família com renda de até 2 salários mínimos e renda média do chefe de família), (5) condições de saúde e idade do chefe de família (taxa de mortalidade por doenças cardiovasculares na faixa etária de 30-
59 anos, mortalidade proporcional antes dos 70 anos, taxa de mortalidade em menores de 5 anos de idade e proporção de chefes de família com 10 a 19 anos de idade). Baseado no consenso de um conselho de experts, cada componente contribui com pesos distintos para o cálculo final do IVS; para maiores detalhes ver publicação anterior 5 .

Com base no valor da soma dos componentes do IVS, os setores censitários foram classificados como de risco baixo, médio, elevado ou muito elevado. As áreas de risco baixo foram aquelas com valores inferiores à média $(2,83)$ menos 0,5 desvio-padrão. As com valores iguais à média $(2,83) \pm 0,5$ desvio-padrão foram classificadas como de risco médio. Áreas com valores superiores a este até o limite de um desvio-padrão foram classificadas como de risco elevado, e aquelas com valores superiores a este foram definidas como áreas de risco muito elevado. Para o presente trabalho, as áreas de risco elevado e muito elevado foram agrupadas para aumentar o poder estatístico da análise.

\section{Inquérito de Saúde da Região Metropolitana de Belo Horizonte}

Os participantes do inquérito de saúde foram selecionados por meio de amostra probabilística em dois estágios. Os setores censitários dos municípios que compõem a Região Metropolitana de Belo Horizonte foram a unidade primária de seleção e o domicílio foi a unidade amostral; todos os residentes no domicílio com 20 ou mais anos de idade foram elegíveis para o estudo, que foi realizado por meio de entrevista face a face. A pesquisa foi realizada em 5.922 domicílios, correspondendo a $79 \%$ do total da amostra; a proporção de perdas foi próxima à estimada para o cálculo amostral (20\%). Todos os participantes residentes no Município de Belo Horizonte com 60 ou mais anos de idade $(n=1.155)$ foram selecionados para o presente trabalho. Maiores detalhes podem ser vistos em outra publicação 7 .

O Inquérito de Saúde da Região Metropolitana de Belo Horizonte foi aprovado pelo Comitê de Ética do Centro de Pesquisas René Rachou da Fundação Oswaldo Cruz, Belo Horizonte, Minas Gerais.

\section{Variáveis do estudo}

A variável dependente do estudo foi o estrato de risco da área de residência, baseado no IVS. As variáveis independentes do trabalho foram baseadas nos componentes do envelhecimento ativo, anteriormente descritos ${ }^{1}$, com algumas adaptações. Assim, foram considerados: (1) ambiente social (escolaridade, situação conjugal, satisfa- 
ção com os relacionamentos pessoais, confiança nas pessoas e temor de assalto); (2) ambiente físico (satisfação com o bairro onde mora e barreiras ambientais, tais como impaciência dos pedestres e/ou motoristas e defeitos nos passeios); (3) comportamentos em saúde (tabagismo atual, atividade física no lazer e consumo de legumes, frutas e hortaliças); (4) condições de saúde (autoavaliação da saúde, número de doenças crônicas e capacidade funcional); e (5) necessidades de serviços de saúde (número de consultas médicas e de hospitalização, dificuldades para conseguir consultas médicas ou medicamentos e uso exclusivo do Sistema Único de Saúde - SUS).

A satisfação com o local de moradia foi baseada em oito perguntas acerca de sentimentos em relação ao bairro onde mora e à vizinhança, considerando-se como insatisfeito aquele que referiu este sentimento em pelo menos quatro perguntas 8 . Foi considerado tabagista atual o indivíduo que já fumou pelo menos cem cigarros durante a vida e continuava fumando. Considerou-se exercício insuficiente no lazer o relato de atividades físicas por pelo menos 20-30 minutos, menos de três vezes por semana nos últimos 90 dias. Foi considerado consumo insuficiente de legumes, frutas e hortaliças a ingestão destes alimentos menos de uma vez por dia nos últimos 30 dias. O número de doenças crônicas foi determinado pela história de diagnóstico médico para artrite/reumatismo, câncer, hipertensão, asma/bronquite, diabetes, angina, infarto, outra doença do coração, derrame, doença renal crônica, doença da coluna/costas e depressão. A incapacidade funcional foi definida pelo relato de muita dificuldade ou incapacidade para realizar quatro ou mais entre 15 atividades da vida diária (AVD) e atividades instrumentais da vida diária (AIVD), como previamente descrito ${ }^{8}$. As atividades consideradas no presente trabalho foram: alimentar-se; vestir-se; tomar banho; escovar os dentes, pentear os cabelos ou lavar o rosto; ir ao banheiro; cortar as unhas dos pés; levantarse da cama; caminhar de um cômodo a outro num mesmo andar; subir dez degraus de escada; tomar seus remédios; preparar as próprias refeições; fazer trabalhos domésticos; caminhar dois ou três quarteirões; sair para fazer compras e utilizar transporte público. As dificuldades para conseguir consultas médicas foram definidas pelo relato de dificuldades de acesso ao local do atendimento, existência de filas, carência de recursos financeiros ou problemas para encontrar um médico quando precisa. Dificuldades para a obtenção de medicamentos foram aferidas pelo relato de inexistência do remédio no posto de saúde ou farmácia, pela dificuldade para ir à farmácia ou conseguir quem vá para buscar o medi- camento, ou problemas financeiros para adquirir o medicamento. Foram considerados usuários exclusivos do SUS os indivíduos que relataram não possuir benefício da medicina suplementar (individual ou corporativo).

\section{Análise estatística}

A análise estatística foi baseada em razões de prevalência (RP) estimadas pelo modelo logístico ordinal, para examinar a força das associações entre as variáveis independentes e os três estratos de risco da área de residência. A premissa do paralelismo das RP foi testada por meio do teste deWald. O cumprimento dessa premissa significa que a RP estimada ao se comparar o estrato de baixa vulnerabilidade com os dois estratos superiores é a mesma obtida quando se compara os dois estratos mais baixos com o estrato superior 9; a escolaridade e o escore do ambiente social foram as únicas variáveis que não cumpriram esta premissa e, neste caso, foram estimadas RP separadas para cada estrato de risco da área de residência.

Inicialmente foi examinada a associação entre cada variável independente e os estratos de risco da área de residência ajustada por sexo e idade. A seguir, foi feita a análise multivariada para examinar a associação entre os escores de cada dimensão (ambientes social, ambiente físico, comportamentos em saúde, condições de saúde e uso de serviços de saúde) e o estrato de risco da área de residência, com ajustamentos simultâneos pelas demais. Uma vez que havia colinearidade entre as variáveis que compõem cada dimensão, foram construídos escores de vulnerabilidade, utilizando-se a análise de componentes principais. Todas as variáveis de cada dimensão foram incluídas na estimativa do respectivo escore. Todas as análises foram realizadas utilizando-se os procedimentos do programa Stata versão 10.1 (Stata Corp., College Station, Estados Unidos) para amostras complexas, considerando-se o peso amostral e o delineamento da amostra.

\section{Resultados}

Entre os 1.155 participantes do inquérito de saúde com idade igual ou superior a 60 anos, 1.055 foram incluídos na presente análise; as exclusões foram devidas a dados faltantes em uma ou mais das variáveis consideradas neste trabalho. Participantes e não-participantes eram semelhantes em relação à idade $(\mathrm{p}=0,775)$, ao sexo $(\mathrm{p}=0,652)$ e ao estrato de risco da área de residência $(\mathrm{p}=0,112)$.

A média de idade dos participantes do estudo foi de 70,1 anos (IC95\%: 69,5-70,7), predominan- 
do o sexo feminino (59,5\%; IC95\%: 56,0-62,9). As porcentagens de residentes nas áreas de riscos alto, médio e baixo foram 15\% (IC95\%: 12,9-17,1), 44,6\% (IC95\%: 41,8-47,5) e 30,4\% (IC95\%: 37,8$43,0)$, respectivamente. Outras características dos participantes da pesquisa estão apresentadas na Tabela 1. As características mais freqüentes foram medo de assalto ao sair de casa $(81, \%)$, exercícios físicos insuficientes no lazer $(77,8 \%)$, diagnóstico médico para uma ou mais doenças crônicas $(68,1 \%)$ e consumo insuficiente de frutas, legumes e hortaliças $(65,5 \%)$.

Na Tabela 2 estão apresentados os resultados da análise da associação entre os componentes das dimensões ambiente social e ambiente físico e o estrato de risco da área de residência, ajustados por sexo e idade. Foram observadas associações graduadas e positivas entre maior estrato de risco da área de residência e pior escolaridade, insatisfação com os relacionamentos pessoais, desconfiança da maioria das pessoas, insatisfação com o bairro onde mora, preocupação com a impaciência de motoristas e/ou pedestres ao sair de casa e temor de cair devido a defeitos nos passeios e/ou dificuldades para atravessar as ruas. Idade, sexo e ausência de cônjuge não apresentaram associações estatisticamente significantes com a área de residência.

A Tabela 3 apresenta os resultados da análise da associação entre os comportamentos em saúde, condições de saúde, necessidades de serviços de saúde e estrato de risco da área de residência, ajustados por sexo e idade. Foram observadas associações graduadas e positivas entre maior estrato de risco da área de residência e comportamentos em saúde (insuficiência de exercícios físicos no lazer e consumo insatisfatório de legumes, frutas e hortaliças), pior condição de saúde (pior auto-avaliação da saúde, diagnóstico médico para pelo menos uma doença crônica e inca-

Tabela 1

Características dos participantes do estudo ( $n=1.055)$. Município de Belo Horizonte, Minas Gerais, Brasil, 2003.

\begin{tabular}{|c|c|}
\hline Características & $\%(I C 95 \%)$ \\
\hline \multicolumn{2}{|l|}{ Demográficas } \\
\hline Sexo feminino & $59,5(56,0-62,9)$ \\
\hline Idade $\geq 70$ anos & $46,1(42,7-49,6)$ \\
\hline \multicolumn{2}{|l|}{ Ambiente social } \\
\hline Escolaridade inferior à mediana ( $<4$ anos completos) & $49,1(45,7-52,6)$ \\
\hline Ausência de cônjuge (solteiros, separados ou viúvos) & $45,7(42,3-49,1)$ \\
\hline Insatisfação ou indiferença com os relacionamentos pessoais & $18,6(15,9-21,3)$ \\
\hline Desconfiança da maioria das pessoas & $41,6(38,4-44,8)$ \\
\hline Medo de assalto ao sair de casa & $81,0(78,4-83,7)$ \\
\hline \multicolumn{2}{|l|}{ Ambiente físico } \\
\hline Insatisfação com o bairro onde mora & $15,3(12,8-17,7)$ \\
\hline Preocupação com a impaciência dos pedestres e/ou dos motoristas ao sair de casa & $33,0(29,9-36,1)$ \\
\hline Teme cair devido a defeitos nos passeios e/ou tem dificuldades para atravessar as ruas ao sair de casa & $49,1(45,8-52,5)$ \\
\hline \multicolumn{2}{|l|}{ Comportamentos em saúde } \\
\hline Tabagismo atual & $11,9(9,7-14,2)$ \\
\hline Insuficiência de exercícios físicos no lazer & $77,8(75,0-80,7)$ \\
\hline Consumo insuficiente de legumes, frutas e hortaliças & $65,5(62,5-68,5)$ \\
\hline \multicolumn{2}{|l|}{ Condições de saúde } \\
\hline Auto-avaliação da saúde razoável, ruim ou muito ruim & $47,0(43,7-50,3)$ \\
\hline Incapacidade funcional & $30,5(27,4-33,7)$ \\
\hline Diagnóstico médico para uma ou mais doenças crônicas & $68,1(64,9-71,4)$ \\
\hline \multicolumn{2}{|l|}{ Necessidades de serviços de saúde } \\
\hline Número de consultas médicas igual ou superior à mediana $(\geq 3)$ nos últimos 12 meses & $45,3(41,9-48,6)$ \\
\hline Hospitalização nos últimos 12 meses & $13,6(11,3-15,8)$ \\
\hline Dificuldades para conseguir consultas médicas & $43,8(40,5-47,0)$ \\
\hline Dificuldades para obter medicamentos & $29,3(26,3-32,3)$ \\
\hline Uso exclusivo do Sistema Único de Saúde & $45,6(42,3-48,7)$ \\
\hline
\end{tabular}

IC95\%: intervalo de 95\% de confiança. 
Tabela 2

Associação entre características demográficas, ambiente social e ambiente físico e estrato de risco da área de residência. Município de Belo Horizonte, Minas Gerais, Brasil, 2003.

\begin{tabular}{|c|c|c|c|c|}
\hline Características & $\begin{array}{l}\text { Baixo (\%) } \\
{[n=382]}\end{array}$ & $\begin{array}{l}\text { Médio (\%) } \\
{[n=572]}\end{array}$ & $\begin{array}{l}\text { Alto }(\%) \\
{[n=201]}\end{array}$ & $\mathrm{RP}(\mathrm{IC} 95 \%)$ * \\
\hline \multicolumn{5}{|l|}{ Demográficas } \\
\hline Sexo feminino & 60,7 & 57,5 & 62,0 & $0,98(0,75-1,28)$ \\
\hline Idade $\geq 70$ anos & 49,0 & 43,1 & 47,4 & $0,88(0,68-1,16)$ \\
\hline \multicolumn{5}{|l|}{ Ambiente social } \\
\hline \multirow[t]{2}{*}{ Escolaridade inferior à mediana ( $<4$ anos) } & 39,9 & 50,3 & 70,6 & $1,89(1,41-2,54)$ \\
\hline & & & & $2,92(1,98-4,31)$ \\
\hline Ausência de cônjuge (solteiros, separados ou viúvos) & 44,3 & 43,9 & 54,9 & $1,33(0,98-1,80)$ \\
\hline Insatisfação ou indiferença com os relacionamentos pessoais & 12,8 & 21,2 & 26,2 & $1,89(1,34-2,66)$ \\
\hline Desconfiança da maioria das pessoas & 35,8 & 44,3 & 49,1 & $1,47(1,12-1,92)$ \\
\hline Medo de assalto ao sair de casa & 80,9 & 81,6 & 81,0 & $0,99(0,70-1,40)$ \\
\hline \multicolumn{5}{|l|}{ Ambiente físico } \\
\hline Insatisfação com o bairro onde mora & 8,5 & 16,8 & 28,4 & $2,65(1,80-3,92)$ \\
\hline $\begin{array}{l}\text { Preocupação com a impaciência dos pedestres e/ou dos motoristas } \\
\text { ao sair de casa }\end{array}$ & 26,8 & 34,1 & 45,8 & $1,73(1,29-2,32)$ \\
\hline $\begin{array}{l}\text { Teme cair devido aos defeitos nos passeios e/ou tem dificuldades } \\
\text { para atravessar as ruas ao sair de casa }\end{array}$ & 42,2 & 50,5 & 63,3 & $1,79(1,35-2,37)$ \\
\hline
\end{tabular}

* Razões de prevalência ajustadas por sexo e idade e intervalo de 95\% de confiança (IC95\%) estimados por meio do modelo logístico ordinal.

Tabela 3

Associação entre comportamentos em saúde, condições de saúde e necessidades de serviços de saúde e estrato de risco da área de residência. Município de Belo Horizonte, Minas Gerais, Brasil, 2003

\begin{tabular}{|c|c|c|c|c|}
\hline Características & $\begin{array}{l}\text { Baixo (\%) } \\
{[n=382]}\end{array}$ & $\begin{array}{l}\text { Médio (\%) } \\
{[n=572]}\end{array}$ & $\begin{array}{l}\text { Alto }(\%) \\
{[n=201]}\end{array}$ & $\mathrm{RP}(\mathrm{IC} 95 \%)$ * \\
\hline \multicolumn{5}{|l|}{ Comportamentos em saúde } \\
\hline Tabagismo atual & 10,3 & 11,5 & 17,8 & $1,44(0,92-2,27)$ \\
\hline Insuficiência de exercícios físicos no lazer & 73,5 & 78,5 & 87,6 & $1,70(1,24-2,33)$ \\
\hline Consumo insuficiente de legumes, frutas e hortaliças & 27,4 & 38,5 & 41,9 & $1,63(1,24-2,15)$ \\
\hline \multicolumn{5}{|l|}{ Condições de saúde } \\
\hline Auto-avaliação da saúde razoável, ruim ou muito ruim & 32,2 & 54,0 & 65,6 & $2,73(2,08-3,58)$ \\
\hline Diagnóstico médico para uma ou mais doenças crônicas & 62,5 & 71,5 & 73,1 & $1,52(1,13-2,03)$ \\
\hline Incapacidade funcional & 25,4 & 30,4 & 44,5 & $1,78(1,30-2,45)$ \\
\hline \multicolumn{5}{|l|}{ Necessidades de serviços de saúde } \\
\hline $\begin{array}{l}\text { Número de consultas médicas igual ou superior à mediana } \\
(\geq 3) \text { nos últimos } 12 \text { meses }\end{array}$ & 36,9 & 48,5 & 58,1 & $1,54(1,22-1,95)$ \\
\hline Hospitalização nos últimos 12 meses & 12,7 & 13,18 & 17,14 & $1,22(0,83-1,80)$ \\
\hline Dificuldades para conseguir consultas médicas & 28,5 & 51,6 & 62,0 & $2,78(2,11-3,67)$ \\
\hline Dificuldades para obter medicamentos & 19,9 & 33,3 & 42,6 & $2,20(1,65-2,93)$ \\
\hline Uso exclusivo do Sistema Único de Saúde & 22,1 & 55,7 & 78,4 & $5,61(4,16-7,57)$ \\
\hline
\end{tabular}

* Razões de prevalência ajustadas por sexo e idade e intervalo de 95\% de confiança (IC95\%) estimados por meio do modelo logístico ordinal. 
pacidade funcional), número de consultas médicas igual ou superior a três nos últimos 12 meses (acima da mediana), relato de dificuldades para conseguir consultas médicas, relato de dificuldades para obter medicamentos e uso exclusivo do SUS. O tabagismo e a ocorrência de hospitalizações nos últimos 12 meses não apresentaram associações estatisticamente significantes com o estrato de risco da área de residência.

Os resultados da análise multivariada da associação entre os escores de ambiente social, ambiente físico, comportamentos em saúde, condições de saúde e necessidades de serviços de saúde e estrato de risco da área de residência estão apresentados na Tabela 4. Pode-se observar que todas as dimensões apresentaram associações positivas e graduadas com o estrato de risco da área de residência, mesmo após ajustamen-

Tabela 4

Resultado da análise multivariada da associação entre os escores de ambiente social, ambiente físico, comportamentos em saúde, condições de saúde e necessidades de serviços de saúde e estrato de risco da área de residência. Município de Belo Horizonte, Minas Gerais, Brasil, 2003.

\begin{tabular}{lc}
\hline Características & RP (IC95\%) * \\
\hline Ambiente social & $1,32(1,12-1,55)$ ** \\
& $1,66(1,37-2,00) * \star *$ \\
Ambiente físico & $1,17(1,02-1,34)$ \\
Comportamentos em saúde & $1,23(1,08-1,40)$ \\
Condições de saúde & $1,18(1,03-1,36)$ \\
Uso de serviços de saúde & $1,65(1,45-1,88)$
\end{tabular}

* Razões de prevalência ajustadas mutuamente pelas outras variáveis listadas na tabela e intervalos de 95\% de confiança (IC95\%) estimados por meio do modelo logístico ordinal; ** Compara o estrato de risco baixo aos estratos de risco médio e alto, em conjunto;

*** Compara os estratos de riscos baixo e médio, em conjunto, ao estrato de risco alto.

tos simultâneos pelas demais. Associação mais forte com o estrato de risco da área de residência foi observada para a dimensão necessidades de serviços de saúde. A segunda dimensão mais fortemente associada ao estrato de risco da área de residência foi o ambiente social, com pior desempenho nas áreas de risco elevado, seguida pela de risco médio.

\section{Discussão}

Os resultados deste trabalho revelaram importantes diferenciais intra-urbanos no ambiente social, no ambiente físico, nos comportamentos em saúde, nas condições de saúde e nos indicadores relacionados às necessidades de serviços de saúde entre idosos residentes no Município de Belo Horizonte. Embora o IVS seja construído parcialmente por indicadores da mortalidade precoce e indicadores socioeconômicos da população mais jovem, as áreas com concentração de idosos vulneráveis foram adequadamente identificadas por ele.

No seu conjunto, os indicadores relacionados ao uso de serviços de saúde apresentaram melhor desempenho nas áreas classificadas como de baixo risco. Entretanto, quando analisados separadamente, algumas diferenças foram observadas. O uso exclusivo do SUS foi a variável que apresentou associação mais forte com o estrato de risco da área de residência. A probabilidade dos usuários exclusivos do SUS residirem em áreas classificadas nos maiores estratos de risco foi cinco vezes maior do que a probabilidade da residência nas demais áreas. As queixas para a obtenção de consultas médicas aumentaram no mesmo sentido, assim como o relato de dificuldades para a obtenção de medicamentos. Por outro lado, a proporção de idosos que haviam tido três ou mais consultas médicas nos últimos doze meses aumentou gradualmente das áreas de baixo risco para as de alto risco.

Estudos anteriores têm mostrado que idosos com melhor situação socioeconômica utilizam mais serviços de saúde. Investigação conduzida em uma grande amostra de idosos brasileiros, utilizando dados da Pesquisa Nacional por Amostras de Domicílios (PNAD), mostrou que aqueles no quintil inferior da distribuição da renda familiar per capita tiveram menos consultas médicas nos doze meses precedentes ${ }^{10}$. Resultados semelhantes foram observados na Cidade do México e na cidade de Santiago, no Chile 11. Contudo, na cidade de São Paulo as consultas médicas foram mais freqüentes entre idosos com pior situação socioeconômica 11 .

A SMS-BH desenvolveu o IVS para subsidiar o planejamento das ações em saúde e a alocação de recursos 5, como anteriormente mencionado. A implantação da Estratégia Saúde da Família no município, por exemplo, priorizou as áreas classificadas como de riscos médio, elevado e muito elevado ${ }^{6}$. Além disso, a composição das áreas sob a responsabilidade de cada equipe e de seus respectivos agentes comunitários de saúde também considerou o IVS, tendo a rede assistencial se organizado de forma diferenciada para cada 
estrato de risco 6 . Os resultados do presente trabalho sugerem que essa estratégia foi adequada para aumentar o acesso às consultas médicas, mas não para reduzir as queixas de dificuldades para a obtenção das consultas e/ou de medicamentos.

Todos os indicadores das condições de saúde apresentaram associações com o estrato de risco da área de residência. Entre eles, a auto-avaliação da saúde apresentou associação mais forte, independentemente do sexo e da idade. A autoavaliação da saúde é considerada um dos indicadores mais importantes para definir o estado de saúde da população ${ }^{12}$. A capacidade funcional é uma das expressões mais importantes do envelhecimento ativo. O número de doenças crônicas é uma medida da carga da morbidade e, junto com os anteriores, reflete a demanda por serviços médicos e sociais. Os resultados do presente trabalho mostraram que essa demanda é maior entre os residentes nas áreas com IVS mais alto. Esses resultados são coerentes com observações realizadas em outros países, mostrando que viver numa vizinhança pobre, com privações, se associa à saúde cardiovascular ruim, a maiores taxas de mortalidade, à maior prevalência da depressão e a comportamentos menos saudáveis 13 , assim como com maior prevalência de limitação funcional e pior auto-avaliação da saúde 14 .

No presente trabalho, atividade física insuficiente no lazer e consumo insatisfatório de frutas, legumes e hortaliças, apresentaram prevalências gradualmente mais altas nas áreas com maiores IVS. A atividade física é o fator que prediz de forma mais consistente o envelhecimento saudável, como tem sido observado em diversos estudos de coorte com pessoas idosas 15,16,17. Dietas ricas em frutas, legumes e hortaliças estão associadas à redução da mortalidade, à diminuição do risco cardiovascular e à redução da incidência de diversos tipos de cânceres ${ }^{18}$. A prevalência do tabagismo é geralmente mais alta em pessoas com nível socioeconômico ou escolaridade mais baixa 19 . Neste trabalho, a prevalência de tabagistas idosos foi mais alta nas áreas com estrato de risco mais alto, mas a diferença não foi estatisticamente significante.

Todas as variáveis que compõem o ambiente social apresentaram associações com o estrato de risco da área de residência, exceto o medo de ser assaltado. O sentimento de insatisfação com as relações pessoais e a desconfiança em relação aos outros podem comprometer as relações com a família, amigos e cuidadores, e reduzir a rede de suporte social do idoso ${ }^{20}$. Essa rede é um forte determinante das condições de saúde ${ }^{21}$. Alguns autores sugerem que a confiança, aspecto central do capital social, tem efeitos protetores sobre a saúde e que, em altos níveis, pode melhorar os recursos emocionais e financeiros do idoso 22 . É provável que em áreas urbanas a redução da rede de suporte social aumente a influência de outros fatores sociais desfavoráveis ao estado de saúde da população idosa 21 . Nesse sentido, as áreas com desvantagens concentradas podem se beneficiar da criação de redes informais de suporte e atividades comunitárias.

Os resultados deste trabalho mostraram que a percepção de insegurança, definida pelo medo de ser assaltado, estava amplamente disseminada entre os participantes do estudo, não apresentando relação com o estrato de risco da área de residência. Grupos focais conduzidos em 33 cidades de países desenvolvidos e em desenvolvimento mostraram que, independentemente do nível real de perigo, preocupações quanto à segurança eram comuns a quase todas as cidades, incluindo aspectos como violência, crimes, drogas, presença de moradores de rua em lugares públicos e iluminação urbana 23 . Crime e medo do crime podem contribuir para manter os idosos em casa e associarem-se à diminuição da atividade física e das visitas a amigos, familiares e prestadores de serviço. Além disso, assaltos violentos podem ter conseqüências drásticas para a população idosa, devido à sua vulnerabilidade para traumas e quedas, em função desses eventos 20

Como esperado, a escolaridade também apresentou forte associação com a área de residência do idoso, com predomínio mais acentuado de pior nível de escolaridade nas áreas classificadas como de risco elevado. A escolaridade é um dos determinantes mais conhecidos das condições de saúde. Pessoas com alta escolaridade tendem a adotar comportamentos mais saudáveis e a procurar mais serviços preventivos de saúde 24 , uma vez que, ao possuírem mais informações, tendem a valorizar de forma diferenciada o cuidado com a saúde. Além disso, quanto maior o nível de escolaridade maior tende a ser o nível de renda e, conseqüentemente, melhores as condições de vida e de saúde 11.

Todos os componentes do ambiente físico apresentaram associações graduadas com o risco da área de residência: insatisfação com o bairro, preocupação com a impaciência dos pedestres e/ou dos motoristas e medo de queda devido aos defeitos nos passeios e/ou dificuldades para atravessar as ruas. Pelo nosso conhecimento, não existem estudos epidemiológicos de base populacional que permitam comparar esses resultados com os de outras cidades latino-americanas. O estudo qualitativo conduzido em trinta e três cidades, anteriormente mencionado, mostrou que incômodos decorrentes da impaciência dos 
motoristas de ônibus eram comuns entre os idosos. Essas queixas incluíram impaciência durante o embarque e desembarque e relutância dos motoristas em aceitá-los como passageiros, entre outras. A dificuldade para atravessar a rua em segurança também foi uma preocupação mencionada com freqüência pelos participantes desse estudo 23. Calçadas mal conservadas, ausência de faixas para pedestres e tráfego pesado constituem barreiras para a população idosa 20 . Calçadas cujos designs são orientados para os pedestres se associam positivamente à atividade física e negativamente à obesidade 25 . O Guia Global da Cidade Amiga do Idoso sugere que calçadas adequadas devem apresentar superfícies homogêneas, planas e antiderrapantes, meio-fio rebaixado para nivelamento com as ruas, ausência de obstáculos como camelôs e carros estacionados, além de largura suficiente para permitir as manobras de cadeiras de roda 23 .

O presente trabalho apresenta vantagens e limitações. A principal limitação do estudo é a sua natureza transversal, que não permite estabelecer relações temporais. Dessa forma, não se sabe se as áreas desfavoráveis da cidade contribuíram para a piora das condições de saúde ou se idosos com piores condições de saúde mudaram-se para estas áreas. Por outro lado, esse delineamento foi adequado para estimar diferenciais intraurbanos das demandas para serviços médicos e sociais em uma amostra representativa da população idosa residente em um dos maiores centros urbanos do país.

Cabe salientar que estudos de vulnerabilidade em áreas urbanas são geralmente voltados para problemas específicos, tais como saúde mental, AIDS, catástrofes, fome e estressores ambientais, como ondas de calor ${ }^{26}$. Além disso, as desigualdades sociais em saúde são geralmente mensuradas por meio de indicadores individuais de renda, riqueza ou escolaridade 10,11. Este trabalho foi pioneiro ao avaliar dimensões distintas da saúde da população urbana idosa sob a perspectiva de um indicador composto, que estima a vulnerabilidade, sendo esta mais uma vantagem do estudo.

Concluindo, os resultados deste trabalho mostraram que o IVS foi adequado para identificar diferenciais intra-urbanos de vulnerabilidade do idoso. Esse instrumento parece ser útil para a identificação de áreas com maior necessidade de serviços sociais e de saúde.

\section{Resumo}

Foi examinada a adequação de um Índice de Vulnerabilidade à Saúde (IVS) para identificar diferenciais intra-urbanos de vulnerabilidade da população idosa. Os participantes do estudo $(n=1.055)$ foram selecionados por meio de amostra probabilística de residentes no Município de Belo Horizonte, Minas Gerais, Brasil, com mais de 60 anos de idade (média = 70,1 anos). O IVS é composto por medidas das condições socioeconômicas e por indicadores da mortalidade precoce do setor censitário. Com base no escore do IVS, o local de residência dos participantes foi classificado em estrato de riscos baixo, médio e alto. Os resultados da regressão logística ordinal multivariada mostraram associações graduadas e estatisticamente significantes entre o estrato de risco da área de residência e o ambiente físico e social, condições de saúde, comportamentos em saúde e necessidades de serviços de saúde (razões de prevalência variando entre 1,17; IC95\%: 1,02-1,34 e 1,65; IC95\%: 1,45-1,88). O IVS parece ser um instrumento útil para a identificação de áreas com maior necessidade de serviços sociais e de saúde entre os idosos.

Vulnerabilidade em Saúde; Saúde da População Urbana; Idoso; Serviços de Saúde

\section{Colaboradores}

L. S. Braga, J. Macinko, F. A. Proietti, C. C. César e M. F. Lima-Costa participaram do delineamento do estudo, da análise dos dados, da redação do trabalho e da revisão crítica do mesmo.

\section{Agradecimentos}

Este trabalho foi desenvolvido como parte das atividades do Núcleo de Estudos em Saúde Pública e Envelhecimento da Fundação Oswaldo Cruz e da Universidade Federal de Minas Gerais, integrante das suas atividades como Centro Colaborador da Secretaria de Vigilância em Saúde do Idoso e Epidemiologia do Envelhecimento, do Ministério da Saúde. Os autores agradecem à Dra Maria Cristina de Mattos Almeida pela inestimável ajuda na classificação do estrato de risco da área de residência dos participantes deste trabalho. 


\section{Referências}

1. World Health Organization. Active ageing: a policy framework. http://www.who.int/ageing/publica tions/en (acessado em 11/Abr/2009).

2. United Nations Population Division. World population prospects: the 2008 revision population database. http://esa.un.org/population (acessado em 11/Abr/2009).

3. Galea S, Ahern J, Karpati A. A model of underlying socioeconomic vulnerability in human populations: evidence from variability in population health and implications for public health. Soc Sci Med 2005; 60:2417-30.

4. Prefeitura Municipal de Belo Horizonte. Estatísticas e mapas. http://portalpbh.pbh.gov.br/pbh/ ecp/comunidade.do?app=estatisticas (acessado em 11/Abr/2009).

5. Secretaria Municipal de Saúde de Belo Horizonte. Índice de vulnerabilidade à saúde 2003. http:// www.pbh.gov.br/smsa/biblioteca/gabinete/risco 2003 (acessado em 11/Abr/2009).

6. Secretaria Municipal de Saúde de Belo Horizonte. Organização territorial dos serviços municipais de saúde em Belo Horizonte. Belo Horizonte: Secretaria Municipal de Saúde de Belo Horizonte; 2003.

7. Lima-Costa MFF. A saúde dos adultos na Região Metropolitana de Belo Horizonte: um estudo epidemiológico de base populacional. http://www. cpqrr.fiocruz.br:81/nespe/NESPE_Portuguese/ BH_p.php (acessado em 11/Abr/2009).

8. Ferreira FR, César CC, Camargo VP, Lima-Costa MFF, Proietti FA. Aging and urbanization: the neighborhood perception and functional performance of elderly persons in Belo Horizonte Metropolitan Area - Brazil. J Urban Health 2010; 87: 54-66.

9. Long JS, Freese J. Regression models for categorical dependent variables using Stata. College Station: Stata Press; 2006.

10. Lima-Costa MFF, Matos DL, Camarano AA. Evolução das desigualdades sociais em saúde entre idosos e adultos brasileiros: um estudo baseado na Pesquisa Nacional por Amostra de Domicílios (PNAD 1998, 2003). Ciênc Saúde Coletiva 2006; 11:941-56.

11. Noronha KVMS, Andrade MV. Desigualdades sociais em saúde e na utilização dos serviços de saúde entre os idosos na América Latina. Rev Panam Salud Pública 2005; 17:410-8.

12. Idler EL, Benyamini Y. Self-rated health and mortality: a review of twenty-seven community studies. J Health Soc Behav 1997; 28:21-37.
13. Lang IA, Llewellyn DJ, Langa KM, Wallace RB, Melzer D. Neighbourhood deprivation and incident mobility disability in older adults. Age Ageing 2008; 37:403-10.

14. Smith KV, Goldman N. Socioeconomic differences in health among older adults in Mexico. Soc Sci Med 2007; 65:1372-85.

15. Haveman-Nies A, De Groot LC, Van Staveren WA Dietary quality, lifestyle factors and healthy ageing in Europe: the SENECA study. Age Ageing 2003; 32:427-34.

16. Leveille SG, Guaralnik J, Ferrucci L, Langois JA. Aging successfully until death in old age: opportunities for increasing active life expectancy. Am J Epidemiol 1999; 149:654-64.

17. Strawbridge WJ, Cohen RD, Shema SJ, Kaplan G. Successful aging: predictors and associated activities. Am J Epidemiol 1996; 144:135-41.

18. Tourlouki E, Matalas AL, Panagiotakos DB. Dietary habits and cardiovascular disease risk in middleaged and elderly populations: a review of evidence. Clin Interv Aging 2009; 4:319-30.

19. Silva GA, Valente JG, Almeida LM, Moura EC, Malta DC. Tabagismo e escolaridade no Brasil, 2006. Rev Saúde Pública 2009; 43 Suppl 2:48-56.

20. Lopez R, Goldoftas B. The urban elderly in the United States: health status and the environment. Rev Environ Health 2009; 24:47-57.

21. Fukuda Y, Nakamura K, Takano T. Municipal health expectancy in Japan: decreased healthy longevity of older people in socioeconomically disadvantaged areas. BMC Public Health 2005; 5:65-9.

22. Nummela O, Sulander T, Rahkonen O, Karisto A, Uutela A. Social participation, trust and self-rated health: a study among ageing people in urban, semi-urban and rural settings. Health Place 2008; 14:243-53.

23. World Health Organization. Global age-friendly cities: a guide. http://www.who.int/ageing/en (acessado em 11/Abr/2009).

24. Lima-Costa MFF. Influência da idade e da escolaridade no uso de serviços preventivos de saúde - Inquérito de Saúde da Região Metropolitana de Belo Horizonte, Minas Gerais, Brasil. Epidemiol Serv Saúde 2004; 13:209-15.

25. Clarke P, Alishire JA, Bader M, Morenoff JD, House JS. Mobility disability and the urban environment. Am J Epidemiol 2008; 168:506-13.

26. Delor F, Hubert M. Revisiting the concept of vulnerability. Soc Sci Med 2000; 50:1557-70.

Recebido em 10/Dez/2009

Versão final reapresentada em 18/Ago/2010

Aprovado em 27/Set/2010 